\title{
Modelos de acción colectiva: modelos de cambio social
}

\author{
Andrés de Francisco
}

\section{Resumen}

El autor realiza una crítica inrema de la teoría analítica de la acción colectiva, tal y como fue formulada por Mancur Olson, mostrando uq̣e tanto la racionalidad individual como la colectiva pueden funcionar de forma "perversan o de forma socialmente beneficiosa, dependiendo del contexro. (Redacción)

Palabras clave: acción colectiva, cambio social, bienes públicos, efectos perversos/subproducros.

Abstract. Models of collective action: models of social change

The arricle makes an internal critique of the analytical theory of collective action, as it was established by Mancur Olson. The criticism shows that cither individual or collective racionaliry can work in a "perverse" way or in a socially useful way, depending on conrext. (Redaction)

Key words: collective action, soctal change, public goods, byproducts.

\section{Sumario}

Dos modelos de acción colecriva

Bibliografía

Marx, la acción colectiva de clase y el cam-

bio social

Quisiéramos en este breve trabajo ver cumplidos dos modestos objetivos: uno analítico y otro, si se nos permite decirlo así, hermenéutico. El objetivo analítico consiste en ampliar y en justificar por qué debe ampliarse la tradicional definición de acción colectiva entendida como aquella empresa común dirigida a la obtención de un bien público. Veremos así que surgen dos grandes modelos de acción colectiva que podríamos denominar, respectivamente, modelo de la mano invisible --siguiendo obviamente la metáfora de A. Smith-y modelo de la cooperación o, por seguir con la metáfora, de la mano visible. El 
interés de esta diferenciación es que nos permitirá considerar el supuesto comportamental del egoísmo y la maximización de la utilidad individual desde una nueva perspectiva más rica en matices. El objetivo hermenéutico, por su parte, consiste en mostrar cómo Marx aplicó, a veces de forma innovadora, ambos modelos de acción colectiva para explicar el cambio macrosocial y, muy específicamente, el proceso de transición del capitalismo al socialismo. Que dicha aplicación haya sido consistente es harina de otro costal que tendrá que cerner la marxología contemporánea.

\section{Dos modelos de acción colectiva}

Desde la seminal obra de M. Olson, The Logic of Collective Action ${ }^{1}$, se ha entendido la acción colecriva como acción cooperativa orientada conscientemente a la obtención de un bien público. A su vez, el concepto de bien público, que es un concepto importado de la teoría económica, se define esencialmente por el hecho de que nadie puede ser excluido de su consumo, haya o no cooperado en el proceso de su obtención ${ }^{2}$. Si, por ejemplo, el estado hace carreteras con los impuestos que los ciudadanos solidarios están dispuestos a pagar, nadie podrá impedir que yo pasee mi vehículo por esas carreteras aunque me las haya arreglado para eludir mi responsabilidad para con hacienda. Me estaré beneficiando entonces de un bien público que no he contribuido a producir y me habré convertido en lo que la teoría de la acción colectiva gusta de llamar free rider, esto es, en un francotirador. Pues bien, ante esta jugosa perspectiva, mi particular cálculo racional de costes-beneficios siempre me recordará tentadoramente que lo más inteligente por mi parte es abstenerme de cooperar a la espera de que otos, por los motivos que sean, proporcionen el deseado bien que impune y gratuitamente podré usufructuar. Si partimos del supuesto de esa racionalidad en sentido restringido, es decir, en sentido instrumental y económico, entonces el diagnóstico obligado de la acción colectiva es desde luego el que ya diera Olson, a saber, que la acción colectiva está intrínsecamente abocada al fracaso dado que la lógica maximizadora del free rider tenderá a generalizarse. Como se sabe, la solución que daba Olson a este problema era básicamente su teoría ad hoc de los incentivos selectivos en el caso de los por el llamados grupos latentes.

Valga lo dicho - un apretado resumen de la teoría «económica» de la acción colectiva - como punto de partida de las reflexiones que a conzinuación pretendemos hacer. Éstas se refieren a dos supuestos tácitos, pero fundamentales, de la teoría. El primero es que cuando la cooperación es suficientemente alta (cuando hay, en otras palabras, una masa critica $a^{3}$ ), entonces el bien público está asegurado. El segundo supuesto, complementario del primero, es que la

1. M. Olson, 1965 .

2. La otra característica esencial de los bienes públicos es su individibilidad, perfecta o imperfecra. Al respecto, ff. M. Taylor, 1976 y 1987, cap. 2 en ambos casos.

3. Un excelente tracamicnto de los modelos de masa crítica se encuentra en Thomas C. Schelling, 1989 , caps. 3 y 7 . 
causa del fracaso de la acción colectiva es la propia racionalidad individual entendida como maximización del interés propio. En efecto, el individuo aislado puede tener interés en la obtención del bien, pero paradójicamente su opción más racional e inmediata es abstenerse de cooperar. Dicho de otro modo, la racionalidad económica individual atrapa a los sujetos potencialmente cooperadores en un dilema del prisionero cuya solución de equilibrio es, como se sabe, un resultado subóptimo: todos quieren conseguir el máximo beneficio pero todos salen perdiendo.

¿Son estos supuestos acaso inamovibles? Pensamos que no lo son por la sencilla razón de que ni el principio de la maximización de la utilidad individual vehicula necesariamente el desastre colectivo ni la cooperación -incluso cuando es total- es garantía de la provisión del bien público. Efectivamente, y como contraejemplo de lo primero, podría defenderse que el crecimiento económico o la eficiencia en la asignación de recursos - considerados ambos como bienes públicos - pueden ser el resultado lateral del comportamiento individualmente egoísta en condiciones de mercado perfectamente competitivo. A su vez, un buen contraejemplo de lo segundo sería la denominada paradoja de la austeridad $d^{4}$. Aceptando, en efecto, que una elevada tasa de ahorro agregado puede considerarse un bien público en términos macroeconómicos, lo que ante esto dice la mencionada paradoja es que si todos los individuos en un entorno económico dado deciden cooperar para conseguir la elevación de aquella tasa de ahorro, y todos de hecho ahorran, el efecto emergente último será una contracción de la demanda agregada, una regresión económica y, por lo tanto, un ahorro agregado menor.

Creemos que los contraejemplos recién citados, lejos de ser caprichosos, animan a intentar una definición más abarcante de acción colectiva que no obstante siga respetando la constricción impuesta por el supuesto de racionalidad restringida y siga ciñéndose a la obtención de bienes públicos. En general diremos que hay dos grandes modelos analíticos para explicar la acción colectiva: el modelo de la mano invisible y el modelo de la cooperación. La característica esencial del primero es que en él cada individuo pretende maximizar su utilidad privada pero, al interactuar con los demás, el resultado lateral de la composición de los distintos cursos de acción es un resultado no buscado de las mismas. La característica esencial del segundo modelo es, por el contrario, la subordinación del interés individual al colectivo. Naturalmente, podría replicarse que un modelo de cooperación así escapará casi con toda seguridad al alcance del supuesto de la racionalidad instrumental. Es verdad que si un individuo coopera por altruismo o por razones extrarracionales -verbigracia, por sentido del deber o por conformidad con normas sociales- la teoría económica convencional tendrá dificultades en explicar su comportamiento. Sin embargo, las dos soluciones internas propuestas al problema de la acción colectiva, al problema del francotirador, en ningún momento han prescindido del supuesto de racionalidad en su versión restringida o econó- 
mica. En efecto, tanto la solución olsoniana de los incentivos selectivos como la basada en la iteración del juego del dilema del prisionero propuesta por Axelrod ${ }^{5}$ asumen que los individuos terminan cooperando porque esta es la mejor manera de maximizar su utilidad privada.

Por lo demás, el rasgo que une a estos dos modelos en un patrón común de acción colectiva es la lateralidad de los efectos de la acción. En otros términos, tanto en uno como en el otro, el bien público es un subproducto de algún otro patrón de comportamiento. En el caso del modelo de la mano invisible el resuitado agregado ni siquiera es previsto por los individuos aislados. En el modelo de la cooperación el bien público es el resultado en principio inmediato de dicha cooperación, pero ella misma es un subproducto bien de la incentivación selectiva, según Olson, bien de la confianza y la información que porporciona la repetición del juego del dilema del prisionero, según Axelrod.

Ahora bien, de la mano de la lateralidad que define a ambos modelos podemos extraer de cada uno de ellos dos submodelos, dependiendo de que el efecto lateral en cuestión sea positivo - un bien público- o negativo - un mal público-. Así, la mano invisible puede ser diestra ("virtuosa") o siniestra ("perversan); la cooperación, adecuada o inadecuada. Un ejemplo clásico de destreza de la mano invisible lo ofrece, al menos según la teoría del equilibrio general, el comportamiento de un sistema perfectamente competitivo regido por el mecanismo de mercado. De ello da fe la primera parte del célebre "teorema fundamental de la economía del bienestarn, según el cual todo equilibrio de mercado perfecto es eo ipso un óptimo de Pareto ${ }^{6}$. Mas, según decíamos, la mano invisible puede también ser endiabladamente perversa. Veremos en la segunda parte de este trabajo cómo Marx utiliza este lado perverso de la smithiana mano invisible para explicar el desarrollo del capitalismo. Por su parte, también la cooperación puede ser un medio adecuado para la consecución de un bien público, como puede ser el caso de una huelga para conseguir subidas salariales, pero igualmente puede ocasionar lateralmente el resultado contrario al deseado, como mostró la paradoja de la austeridad descrita más arriba.

En resumen, podemos decir que tenemos dos grandes modelos de acción colectiva de los que se derivan cuatro submodelos según la bonanza o maldad del efecto agregado en cuestión, cuya característica común, insistimos, es su naturaleza esencialmente lateral. La principal conclusión que podemos extraer de esta ampliación teórica es que la racionalidad maximizadora y el egoísmo no tienen por qué ser los causantes del fracaso de la acción o la interacción colectiva. En efecto, en el primer submodelo - el de la diestra mano invisible- producen más bien lo contrario, un beneficio público. Más aún, también podemos decir, tal vez rizando un tanto el rizo, que esa misma racionalidad y ese egoísmo pueden, a su vez, ser efectos de otras variables. Por ejemplo, en el modelo del dilema del prisionero, el verdadero causante del comportamiento egoísta y miopemente maximizador de los individuos atrapados en él es en

5. Cf. R. Axelrod, 1984.

6. Cf. A. Sen, 1987, p. 34-38. 
realidad la desconfianza mutua que se profesan. De hecho, como ya dijimos, si el juego se repite suficientes veces los individuos comprenden que lo mejor es cooperar, lo comprenden cuando han aprendido a confrar mutuamente. Pero la confranza depende del nivel de información de que disponen los contendientes, muy escasa en la primera jugada pero progresivamente creciente con la repetición del juego. En otras palabras, el dilema del prisionero es debido no tanto a que los individuos sean egoístas o racionalmente maximizadores - y podemos asumir, por una simple cuestión de parsimonia metodológica, que lo son-, sino más bien por la escasez de información padecida por los sujetos atrapados en él.

Por otro lado, y esta será nuestra última conclusión analítica, no está del todo claro que el francotirador llegue a serlo por egoísmo, es decir, porque el cálculo costes-beneficios que realiza dictamine la no cooperación como su mejor estrategia. Podría ser que el individuo se abstenga de cooperar por razones no consecuencialistas sino deontológicas. Imaginemos, por ejemplo, que convocamos una huelga en el seno de una fábrica japonesa y que hemos conseguido convencer a la mayoría de que deben apoyarla porque están explotados: la huelga es mayoritariamente secundada. ¿Diremos entonces que la minoría de esquiroles es víctima del egoísmo, que no cooperan porque así maximizan su beneficio privado? No necesariamente, pues podrían tener razones deontológicas que les impidieran participar en la actividad huelguística, por ejemplo, un especial sentido de fidelidad y deber hacia el patrón, etc. Dicho de otro modo, no todo nocooperador tiene necesariamente que ser un francotirador, aunque termine beneficiándose de un bien público que no ha contribuido a producir.

\section{Marx, la acción colectiva de clase y el cambio social}

La teoría marxiana de la historia -el materialismo histórico- es una teoría del cambio macrosocial. Su objetivo es explicar por qué y bajo qué condiciones los modos de producción son sustituidos por otros más aptos para el ulterior desarrollo de las fuerzas producrivas ${ }^{7}$. Sin embargo, el modo de producción que mayor atención recibió de Marx fue el capitalista. El materialismo histórico aplicado al capitalismo aspira a explicar el proceso escructural de cambio dentro de éste y el proceso de transición hacia un modo de producción "superior", el socialista. Pues bien, lo que ahora queremos subrayar es que los modelos explicativos que Marx utiliza para dar cuenta de ambos tipos de cambio social son harto distintos. Mientras que utiliza un modelo de explicación de la mano invisible en el primer caso - el del cambio dencro del capicalismo-, recurre a un modelo de cooperación o de mano visible -mla lucha de clasespara explicar el proceso de transición del capitalismo al socialismo ${ }^{8}$. Veámoslo con algún detenimiento.

7. Sobre la teoria de la historia de Marx, cf. G. Cohen, 1986 y 1988, y J. Elster, 1985, especialmente la parte II. 5 .

8. Cf. G. Herne, 1975 , p. $534-535$. 
Los modelos de la mano invisible pertenecen a la subvariedad supraintencional de la explicación causal. Esto no quiere decir más que lo que ya apuntábamos en la sección anterior: que los resultados agregados de la acción intencional son ellos mismos no intencionados ${ }^{9}$. La apelación a este tipo de explicaciones supraintencionales convierte a Marx, como no se ha cansado de defender el destacado marxólogo Jon Elster, en pionero de la moderna metodología de las ciencias sociales ${ }^{10}$. Mas, según decíamos antes, la mano invisible puede ser virtuosa o perversa; lo que da pie a una reflexión de carácter histórico.

Sabido es, en efecto, que la metáfora de la mano invisible constituye una tópica de la Ilustración: así Mandeville con su uPrivate Vices, Publick [sic] Benefits", así también Kant con sư "ungesellige Geselligkeit», así incluso Hegel con su "List der Vernunft". En todos ellos el argumento es el mismo aunque, qua ilustrados, confiaran con optimismo en la eficaz destreza de la mano invisible para conseguir resultados siempre beneficiosos, no importando que los medios (el vicio privado, la insociabilidad o la irracionalidad) carecieran de la dignidad de los fines. Marx mantiene una relación dual respecto a esta herencia ilustrada $y$, como ha recordado recientemente Hirschman, mefistofélica ${ }^{1 \mathrm{t}}$. Es verdad, en efecto, que incorpora a su análisis del capitalismo este tipo de explicación basada en el postulado de una mecánica social. Sin embargo, en Marx cabría diferenciar dos planos: uno microdinámico (científico-concreto) y otro macrodinámico (histórico-filosófico genérico). Es así que -como buen discípulo de Hegel que siempre fue ${ }^{12}$ - desplaza el optimismo ilustrado al plano macrodinámico (la historia, globalmente considerada, avanza por el lado malo, mefistofélicamente, pero promete un final feliz) al tiempo que hace uso de las explicaciones de la mano invisible en el plano microdinámico (el del análisis concreto del capitalismo), pero invirtiéndoles el signo, esto es, vaciándolas del optimismo dieciochesco, cabría decir, des-ilustrándolas. Así recurre por lo normal a estas explicaciones para demostrar los efectos agregados perjudiciales de la acción individual en el seno de la sociedad civil burguesa, para captar las externalidades negativas de un sistema de mercado basado precisamente en la maximización de beneficio privado. Es lo que Elster, siguiendo a Sartre, denomina fenómeno de la contrafinalidad ${ }^{13}$. En Marx hay múltiples análisis que responden a este patrón explicativo, pero tal vez sea paradigmático el análisis de la tendencia a la baja en la tasa de ganancia, uno de los pilares por lo demás de su explicación de la evolución del sistema capitalista, en la que son justamente las innovaciones técnicas (perfectamente racionales desde la lógica maximizadora de los productores que compiten individualmente en el mercado) las

9. Cf. J. Elster, 1983, "Introducción" y I.1.

10. Cf. J. Elster, 1985, cap. I y 1991. cap. 2.

11. Hirschman (1991, p. 14), naturalmente, se refiere a la interpretación que Goethe ofrece del quehacer de Mefisto, como unna parte de esa fuerza que siempre quicre el mal, pero siempre produce el bien".

12. Para una, a nuestro juicio, inapelable demostración del hegelianismo del Marx maduro, $c f$. M. Sacristán, 1983, p. 317-367.

13. J. Elster, 1985 , p. 25. 
que provocan aquella reducción tendencial del beneficio. Pero el mismo patrón explicativo - el de la perversidad de la mano invisible- se repite, esta vez en un plano sociológico, cuando Marx afirma, en el Manifiesto del Partido Comunista, que el ascenso social de la burguesía va acompañado del nacimiento de una nueva clase social destinada a derrocarla, el proletariado ${ }^{14}$.

No deja de resultar curioso que lo que Hirschman ha llamado tesis de la perversidad constituya un rasgo central de la "retórica de la reacción", esto es, del pensamiento conservador ${ }^{15}$. Y decimos que resulta curioso ya que la explicación marxiana del desarrollo del capitalismo se basa precisa y esencialmente en ella. ¿Constituye dicha tesis acaso el punto donde ambas retóricas se tocan, la de la reacción y la de la revolución? Como pensamos que sería absurdo confundir a Marx con un Burke o un de Maistre, hemos de cualificar su uso de la tesis de la perversidad, tesis con un cariz indudablemente conservador. Pues bien, dicha cualificación viene servida por nuestra anterior distinción de planos en el análisis marxiano del capitalismo. En efecto, un sistema de mercado basado en el imperativo de la acumulación y valorización constantes del capital es un sistema para Marx perverso, esto es, que produce externalidades negativas, y las produce no sólo en sentido socioeconómico — generando ineficiencia económica y conflicto social-, sino también en sentido ético-normativo -generando alienación y explotación-. Si la crítica de Marx al capitalismo se hubiera quedado ahí -en el plano microdinámico-, entonces sería fácil emparejarla con la abundante producción romántica y reaccionaria del siglo XIX. Sería una postura meramente reactiva ante el definitivo hundimiento de una idealizada sociedad premoderna, de una primitiva Arcadia feliz. Pero ocurre que Marx siempre acentuó el carácter progresivo del capitalismo. En otras palabras, la alienación y la explotación eran para él el precio que habia indefectiblemente que pagar, situados ahora en un plano histórico-filosófico, para que la historia realizara "dialécticamente" su cometido: dicho en términos hegelianos, llevar a la humanidad al lugar donde realidad y racionalidad fueran de consumo. A esto Marx le dio el nombre de comunismo. Pero la historia habría de valerse de su Mefistófeles.

El Mefistófeles de Marx no fue otro que el modo de producción capitalista que, si en el plano microdinámico quiere el bien y opera el mal, en el plano histórico-filosófico persigue el mal pero promete el bien ${ }^{16}$. En definitiva, podemos decir que es el hegelianismo que nunca abandona Marx el que le permite hacer uso de la tesis de la perversidad sin caer por ello víctima de la retórica de la reacción.

Sin embargo, Marx no se limitó a elaborar una teoría del cambio dentro del capitalismo, sino que también adelantó una teoría de la transición socialista. Y como hemos venido anticipando, el modelo explicativo que utiliza en

14. Cf. K. Marx-F. Engels, 1847-8, p. 27 y s.

15. Cf. A. Hirschman, 1991, cap. 2.

16. Un texto maravilloso e instructivo sobre la filosofia de la historia de Karl Marx es el de G. Cohen, 1974. 
este caso es un modelo, vale decir, de mano visible, esto es, un modelo de cooperación. Nos referimos a su teoría de la consciencia y la lucha de clases, consciencia y lucha que se fraguan sobre el terreno abonado por el desarrollo interno del capitalismo, que -insistimos una vez más-- Marx intenta captar con el modelo de la perversa mano invisible. En otro lugar hemos analizado detenidamente los límites del modelo de cooperación aplicado por Marx a la lucha de clases ${ }^{17}$. Por ello, aquí bastará con que apuntemos rápidamente dos cosas. En primer lugar y fundamentalmente, Marx fue consciente del problema interpersonal de la acción colectiva que se presentaba a la clase obrera, dado que tuvo en cuenta que los obreros también podían competir entre sí, poniendo en jaque la unidad misma de la clase. Pero, en segundo lugar y no menos fundamentalmente, Marx no supo resolver analíticamente el problema de la cooperación intraclasista. En efecto, se limitó a interponer condiciones muy genéricas y poco discriminatorias de tal manera que la lucha de clases quedaba teóricamente indeterminadas el proletariado, esto es, podía cooperar entre sí contra la burguesía tanto como podía cooperar con elía enfrentándose entre sí. Ninguna posibilidad quedaba excluida. Esas condiciones generales eran fundamentalmente de dos tipos: de carácter cognitivo y de carácter socioeconómico. Las primeras tienen que ver con la consciencia de clase y con la correcta identificación del enemigo de clase. Pero en ningún caso puede afirmarse que sean condiciones suficientes para la cooperación de clase. En efecto, el fantasma del francotirador puede minar también la cooperación en un colectivo con consciencia, por ejemplo, de que es injustamente explotado por otro colectivo. Por otra parte, la correcta identificación del enemigo parece más bien una condición del éxito de la acción estratégica en general - colectiva o no-, pero de nada servirá si por acaso la lógica del free riding llega a contaminar al grupo en cuestión. El otro tipo de condiciones generales interpuestas por Marx podrían considerarse como condiciones estructurales de coyuntura socioeconómica. Nos referimos fundamentalmente a la tesis de la polarización radical de la riqueza y extrema pobreza de la clase obrera ${ }^{18}$. Bien es cierto que estas condiciones son considerablemente restrictivas, mas parece probado que las tres gtandes revoluciones "modernas" conocidas (francesa, rusa y china) fueron alumbradas por una profunda crisis económica que explicaría la aparición de procesos de insurgencia (urbana o campesina) desde abajo. Ahora bien, según ha defendido recientemente Theda Skicpol, dichos procesos insurgentes sólo se convertirán con éxito en revoluciones - como quería Marx- si se cumplen adicionalmente ciertas condiciones estructurales de coyuntura sociopolitica $^{19}$. Es el caso, sin embargo, que Marx no supo dar con estas últimas. En resumidas cuentas, si bien Marx detectó correctamente el problema interno de la acción colectiva, su modelo de cooperación - del que se valió para dar cuenta del proceso de transición del capitalismo al socialismo-- quedó pen-

17. Cf. A. de Francisco, 1992, p. 166-179.

18. Cf. K. Marx-F. Engels, 1966, p. 35.

19. Cf. Theda Skicpol, 1979. 
diente de una adecuada elaboración teórica. Y ello independientemente de la consistencia teórica con el modelo de la perversa mano invisible con el que intenta explicar el otro gran proceso de cambio social, el del desarrollo interno del capitalismo.

\section{Bibliografía}

AXEIROD, R. (1984). The Evolution of Cooperation. Nueva York: Basic Books [La evolución de La cooperacion. Madrid: Alianza, 1986].

Cohen, G. (1974). "Marx's Dialectic of labour". Philosophy and Public Affairs, 3, núm. 3, p. 235-261.

- (1986). La teoria de la historia de Karl Marx: una defensa. Madrid: Siglo XXI-Pablo Iglesias.

- (1988). History, Labour and Freedom (Themes from Marx). Oxford: Clarendon Press.

DE FRANCISCO, A. (1992), "Qué hay de teótico en la "teoria" marxista de las clases?". Zona Abierta, 59-60, p. 157-186.

EISTER (1983). Explaining Technical Change. Cambridge: Cambridge University Press.

- (1985). Making Sense of Marx. Cambridge-París: Cambridge University Press/Editions de la Maison des Sciences de l'Homme.

- (1991). Una introducción a Karl Marx. Madrid: Siglo XXI.

Hernes, G, (1975). "Structural Change in Social Processes". American Journal of Sociology, vol. 82, núm. 3, p. 513-547.

Hirschman, A.O. (1991). The Rhetoric of Reaction. Cambridge, Mass.: The Belknap Press of Harvard University Press.

MarX, K. y Exgels, F. (1845). La Ideología Alemana. La Habana: Edicion Revolucionaria, 1966.

- (1847-8). Manifiesto del Partido Comunista. Barcelona: Akal, Obras Escogidas, vol. $1,1975$.

Oi.son, M. (1965). The Logic of Colective Action (Public Goods and the Theory of Groups. Cambridge, Mass: Harvard University Press.

Ovejero, F. (1989). Intereses de todos, acciones de cada uno. Madrid: Siglo XXI.

SACRISTÁN, M. (1983). Sobre Marx y marxismo (panfletos y materiales I). Barcelona: Icaria.

SCHELling, Th.C. (1989). Micromotivos y macroconductas. México: FCE.

SEN, A. (1987). On Ethic and Economics. Oxford: Basil Blackwell.

SKOCPOL, Th. (1979). States and Social Revolutions: Comparative Analysis of France, Russia and China. Cambridge: Cambridge University Press.

TAYLOR, M. (1976). Anarchy and Cooperation. Londres: Wiley.

- (1987). The Possibility of Cooperation. Cambridge: Cambridgc University Press. 